\title{
Decentralized planning for the Ganga Basin. Decomposition by political units
}

\author{
GEORGE KARADY and PETER ROGERS* \\ Temple, Barker and Sloane Inc., 33, Hayden Avenue, Lexington, Massachusetts 02173, USA \\ *Department of Environmental Engineering and City Planning, Harvard University, Pierce \\ Hall, 121, Cambridge, Massachusetts 02138, USA
}

\begin{abstract}
In this study the greater Ganga Basin is decomposed by political units for analysis instead of by hydraulic sub-basins. This method is more logical because water-resources-use planning is under the individual state jurisdiction but the Central Government is responsible for meeting the low flow requirement at Farakka. Maximisation of national benefits is taken in terms of total area irrigated. The solution technique proposed is an iterative procedure.

The problem is analysed under two schemes (i) flow quota scheme and (ii) resource allocation scheme. These schemes are worked out by three algorithms. The first algorithm requires that minimum flow at any point in the Ganga should be greater than the sum of the minimum quota fixed by the central Government for all the states above that point. Under algorithm two, water that leaves an upstream state may be used by a downstream neighbour provided that this neighbour allows a flow greater or equal to the sum of its and all the upstream states quota to pass into the next state. In algorithm three the restriction of algorithm two has been lifted. The merits and demerits of each algorithm are discussed.
\end{abstract}

Keywords. Large scale systems; decomposition; decentralized planning; flow quota scheme; resource allocation scheme.

\section{Introduction}

The purpose of this study is to apply a decentralized planning solution technique to the coordinating-screening Ganga river basin model developed by Chaturvedi et al (1985). Their large, deterministic linear programming model, which describes in detail the water in the Ganga basin, was solved in its entirety. Later, a decomposition by river basin solution was proposed by Rogers \& Kung (1985). This latter approach assumes the existence of river basin authorities, which solve their own, much smaller resource allocation problems and whose action is coordinated by some central planning agency. The method presented here addresses the more complicated but realistic issue of decomposition by provinces or states. The motivation for such a scheme is as follows. Water resources use planning is under the jurisdiction of individual states, but it is the central government which is responsible for meeting the low flow requirements at Farakka. The central government's (cG) objective is presumably to meet this above constraint while coordinating the state governments' (SG) work in such a way that the total national benefits, in our case rabi and kharif season irrigation of agricultural land, are maximized.

The technique proposed is an iterative one, in which the cG decrees some initial allocation of water for each state so that the low flow constraints are met at Farakka. The states solve their own, pertinent water allocations and report back to the cG some shadow price on the initial allocation. The cG then reallocates in such a way that there is 
a net increase of total area irrigated in the states. This back and forth exchange of information goes on until the $\mathrm{CG}$ cannot engineer a further improvement in its objective. Hopefully, as was the case in the basin-wise decomposition, the iterative process will terminate in a few steps. These iterations are equivalent to "rounds of discussions" between the CG and the sG, each of which has conflicting goals and is trying to secure the best possible solution for itself.

One of the broad schemes proposed calls for the CG to ask each SG to contribute a certain amount of water to the national and international cause of satisfying the low flow requirements at the Bangladesh border. Apart from this intervention the cG allows the states to use their water resources in the most efficient way possible. The states do this by solving their water allocations subject to the CG imposed quota and to other minimum flow requirements negotiated among themselves.

A second scheme, which will henceforth be referred to as the "resource allocation scheme" requires that the CG tell the states how much water they can divert for irrigation in each season. Under this proposal the CG must be aware of the hydrological inputs to each of the states, so that it can check that the states do not misrepresent the quantity of water they use. The advantages and disadvantages of this latter decomposition method will be further expounded below.

The major assumptions and simplifications made to the coordinating-screening model are restated as follows: (i) The objectives are to maximize rabi and kharif season irrigation subject to the constraint that during one month of the summer season, the same area be irrigated as during the kharif season (this summer irrigation is to prepare the soil for sowing), (ii) no consideration is given to power generation.

Other simplifications and their justifications are presented in $\S 4$. A simplified view of the Basin considered as 6 states plus Bangladesh is shown in figure 1.

\section{Description of the decomposition algorithms}

Each of the algorithms considered is of the primal feasible type, for it was deemed important to be able to stop the process, if necessary, before the optimal solution was found and still end up with a feasible set of operating rules. A further requirement was that the operating policy be relatively easily implemented and enforced.

The greatest difficulty in connection with the decomposition by state scheme was dealing with the serially-coupled nature of the problem. If a state decides to use less water, i.e. allow more to pass into the downstream states, the question arises as to what the optimal strategy is for the use of the extra water by these states. The algorithms presented attempt more or less successfully to decouple the states from each other. The situation is further complicated by the fact that the model considers not one, but three, seasons among which there is also considerable coupling. For this reason at each iterative step only one parameter of one season is changed.

\section{Flow quota scheme}

Under this scheme, each state contributes a certain amount of water, set by the CG, to the quantity required at Farakka. The cG communicates an initial quota, $F_{N j}^{(0)}$, (where $N$ refers to one of the states, $j$ refers to the season) to each of the state governments. The SG solves its problem, and returns shadow prices on its input and output constraints, as 


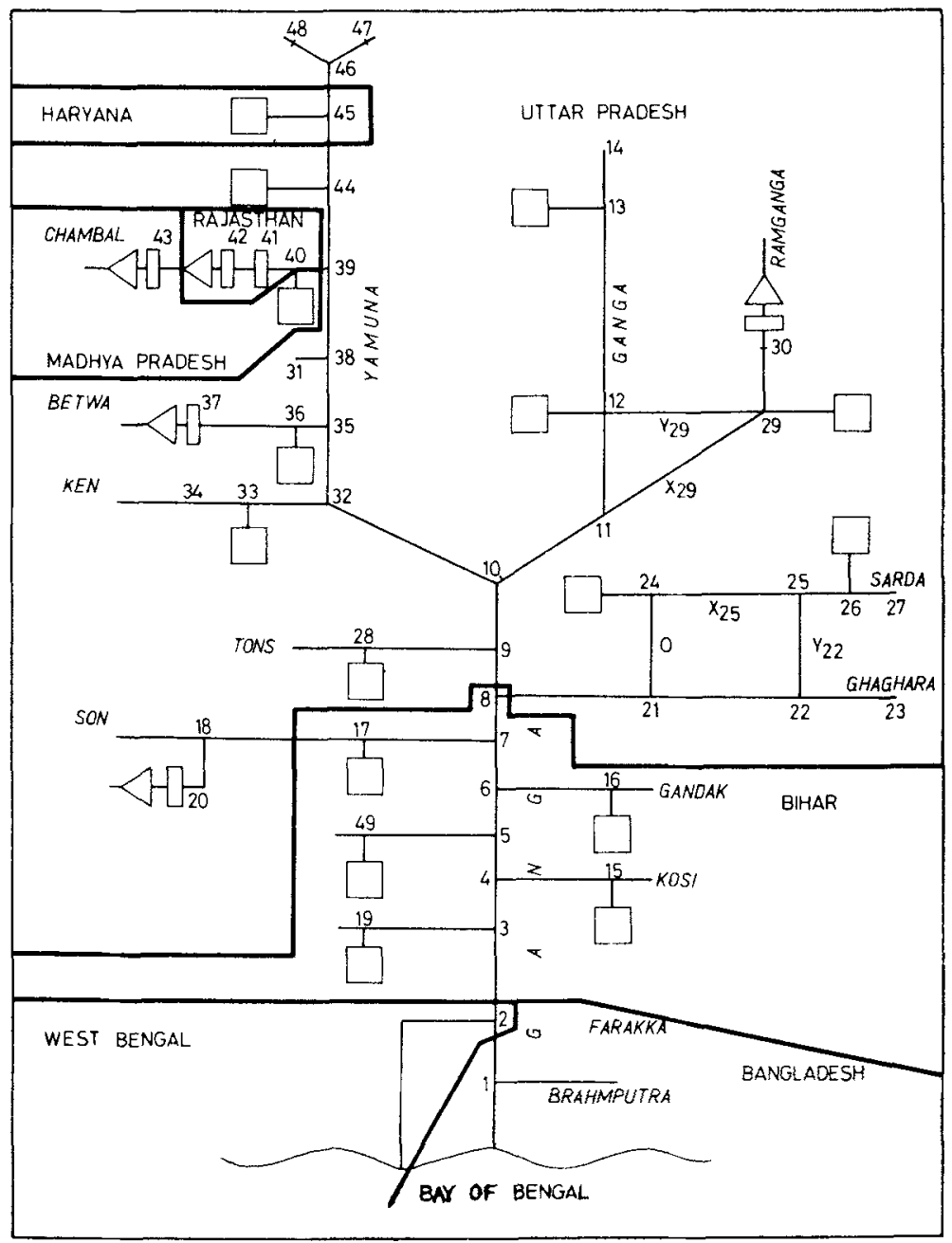

Figure 1. Line diagram of the Ganga basin

well as the range of the relevant right hand sides, over which the shadow prices are unchanged, to the CG. The CG reassesses the data and changes the $F_{N j}^{(k)}$, where $k$ is the iteration number, in such a way that the sum total of the irrigated area in the states increases. This usually means that states with a high shadow price for water are required to contribute less, while states with a low shadow price for water are to contribute more to the common cause. The iterations are carried out until one of the following criteria is met: (i) all shadow prices are equal; (ii) further increase or decrease of some of the $F_{\mathrm{Nj}}^{(\boldsymbol{k})}$ would result in system infeasibilities.

A block diagram of this scheme is shown in figure 2 along with arrows depicting the information flow between CG and SG. The CG sets the $F_{N j}^{(k)}$ and enforces or at least checks them by occasionally measuring the actual flow of water across the states' borders. The SG relays information concerning the shadow prices on its input and output of water and the right hand side ranges over which these shadow prices are constant. Information as to 


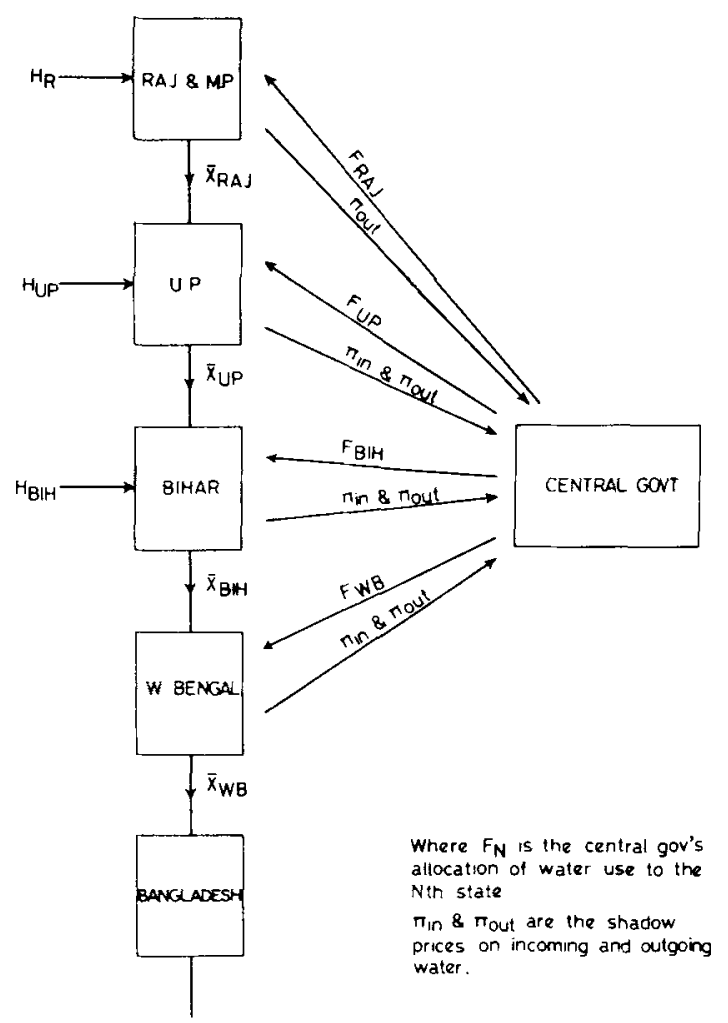

Figure 2. Schematic diagram and information flow between Central Government and State Government.

each state's optimal irrigation for a given $F_{N_{j}}^{(k)}$ is also released to the cG. This is the extent to which information is exchanged among the different hierarchies of government.

Unfortunately, the algorithms considered below are very much subject to misrepresentation by the SGS. While the CG can ascertain, by direct measurement, that each state allows at least $F_{N j}^{(k)}$ amount of water to leave its territory, it cannot be sure whether the shadow prices or ranges on their right hand sides have been falsified or not. In the analysis that follows, it is assumed that the state governments do not misrepresent these data. The problem of the $\mathrm{CG}$ in finding the optimal $F_{N_{f}}^{(\boldsymbol{k})}$ when misrepresenting is considered should be investigated at a future date, for it may represent a more realistic situation than the one considered here. It should be noted that the cG possesses no direct mechanism by which it could force the states to comply with the required $F_{N j}^{(k)}$, should their output fall short of this targeted value.

\subsection{Algorithm I}

Complete decoupling of the states, if not of the seasons, may be achieved by requiring that the minimum flow anywhere in the main stream of the Ganga be greater than the sum of the $F_{N j}$ 's of all of the upstream states. See figure 3a for illustration. This is a very restrictive constraint, for under this scheme downriver states can in no way utilize the water released upstream. The system is effectively separated into one similar to that 


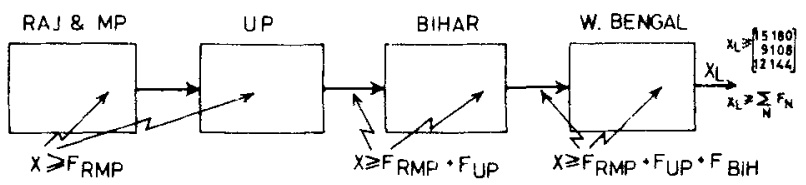

(a)

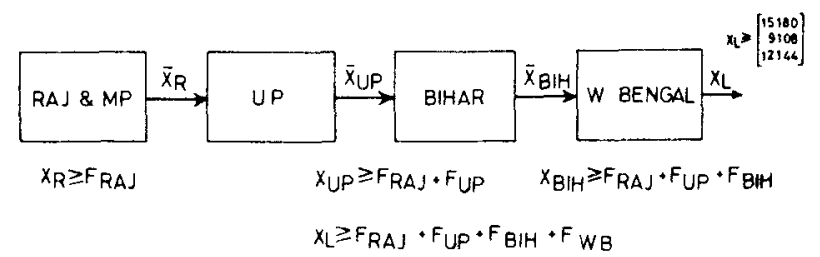

(b)

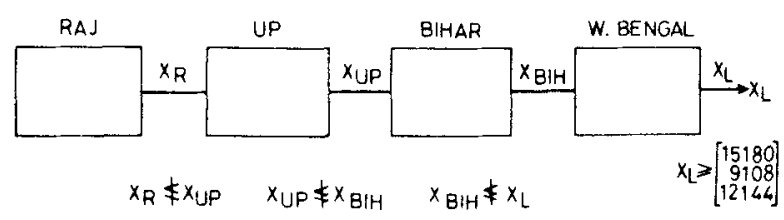

(c)

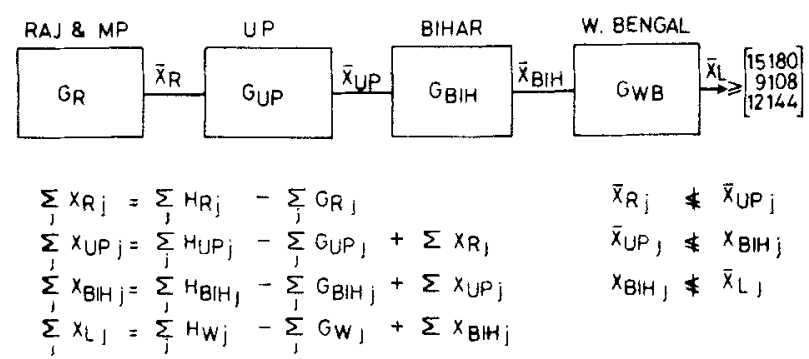

(d)

Figure 3. a. Outflow quota scheme, algorithm I. b. Outflow quota scheme, algorithm II. c. Low flow quota scheme, algorithm III. d. Resource allocation algorithm.

considered by Rogers \& Kung (1985) in their basinwise decomposition. They showed that convergence was rapid; only three iterations were required. From a practical, implementational point of view, algorithm $I$ is infeasible, for it requires that the flow of water be regularly checked at each main stem node, and the final solution may be socially inefficient, since it could allow a situation in which Northern uP is underirrigated, but Southern up has too much water. If the above constraint were relaxed within UP and were only enforced at its border with Bihar, UP may be able to increase its irrigated areas considerably. These considerations bring us to a second, more realistic algorithm.

\subsection{Algorithm II}

Under this algorithm, water that leaves an upstream state may be used by its downstream neighbour provided that this neighbour allows a flow greater or equal to the 
sum of its and all the up-stream states' $F_{N j}$ to pass into the next state. The situation is illustrated in figure $3 \mathrm{~b}$. While the algorithm may lead to higher overall irrigation levels, it also poses some major computational difficulties. Monotonic convergence to the global optimum is not assured anymore. It also becomes difficult to ascertain whether after a seemingly last iteration a global optimum has in fact been attained. At the heart of these difficulties lies the fact that the ranges on the right hand sides are computed assuming that only that this side is changed. This means, for example, that one either changes the inflow or the outflow constraints of a state. Changing both or changing one in more than one season may lead to erratic results because a shift in one of the constraint's right hand side may lead to a changed shadow price or range for another constraint. With all this in mind, consider the situation encountered in the first computational example after the ninth iteration. The status of the model after this iteration is shown in figure 4a. Briefly then, the shadow prices indicate that it would be beneficial to increase either the input to UP and/or to reduce its output to Bihar. Neither of these possibilities is open to the CG, for UP's output already equals Rajasthan's quota with $F_{U P j}=0$ in the first season. Is one at an optimum? In a sense a local optimum has been reached, but can a still higher optimum be reached by first increasing up's output (a sub-optimal move) and then increasing the input to it from Rajasthan? Will such a move lead to a better solution, and if yes, by how much should one increase up's output? These questions may only be answered by actually performing the calculations. It is because of considerations like these that the convergence is not monotonic and is heavily dependent on the 'path' or decisions that were taken by the co to get to the 'local maxima'. At each iteration the CG chooses from among a number of decisions, all of which will improve the overall solution. It does not know a priori which of the choices will take it the "farthest", along the decision tree. It may have to decide to "skip from one branch to another" in a sub-optimal iterative step in order to progress further towards the global optimum. The fifth iterative step was such a 'skipping' move. See figure 5 for further details.
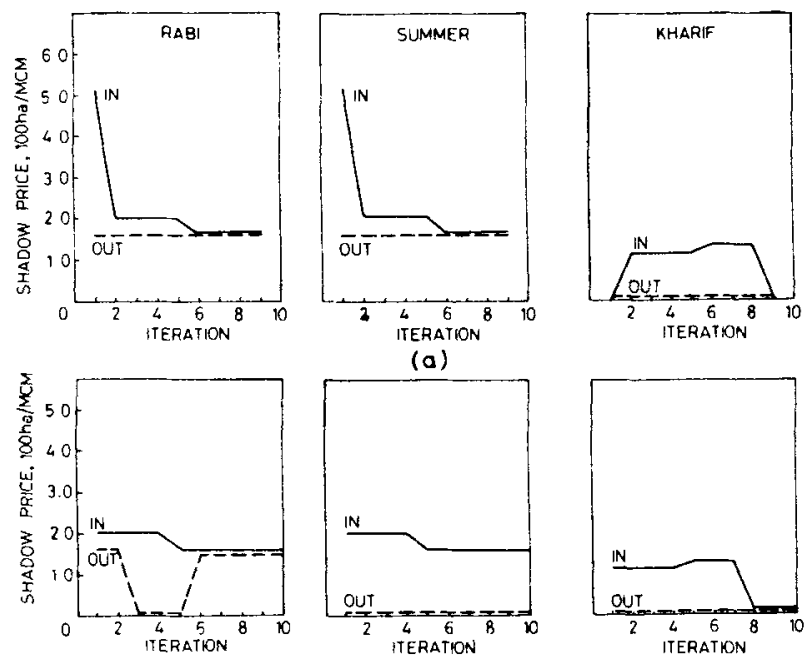

(b)

Figure 4. Shadow prices for Uttar Pradesh. a. Algorithm II. b. Algorithm III. 
In defence of the algorithm, it must be pointed out that there was very little improvement to the objective function value after the fourth iteration. Familiarity with the system would also tend to make one doubt that the solution after the ninth iteration may be significantly improved. Nevertheless, it is entirely plausible that the relative success of algorithm II on the Ganga basin problem is due to the insensitivity of the model to changes in the input and output parameters. In the light of these difficulties, one ought to exercise great caution when applying the algorithm to an unknown general system.

\subsection{Algorithm III}

In the second algorithm, Rajasthan's quota, for example, could be used by uP but had to be accounted for at its border with Bihar. In the algorithm considered here, this restriction is lifted. The $F_{N j}$ 's are no longer the $N$ th state's contribution to the quota to be met at Farakka, but it is the actual amount of water the $N$ th state is required to release to its neighbour. In effect the CG is not only seeking the best solution subject to the Farakka constraint, it also takes upon itself to redistribute the water of the Ganges among the six states in such a way as to maximize the sum of the areas irrigated in all of the states. Because this algorithm is less restrictive than algorithms I and II, one expects it to yield the highest solution of the three. Figure $3 \mathrm{c}$ illustrates the situation. While this method suffers from fewer computational difficulties than the second algorithm, one wonders whether or not it constitutes a grave infraction on the part of the $\mathrm{CG}$ on the states' sovereignity, to dictate the redistribution of their resources in such a manner.

This third algorithm converges monotonically to what is believed to be the global maximum for the system. As yet, this statement has not been proved. The path to the maximum does not seem to be unique, just as the solution, as far as the decision variables are concerned, is not unique.

In summary then, algorithms were presented for the decomposition by state of the Ganges basin. All the three are susceptible to misrepresentation by the sGs. Algorithm I, which is the least difficult to solve, is almost impossible to implement in practice. Algorithm II, while most palatable in a political sense (contributions to a national cause) has difficulties in converging to a global optimum. Algorithm III performs well mathematically, but may promote animosity between the states and the CG.

\section{Resource allocation schemes}

Instead of prescribing the low flow requirement $F_{N j}$, the CG allocates a certain amount of water $G_{N j}^{(k)}$ to be used by the $N$ th state in the $j$ th period. The explicit form this constraint takes is

$$
\sum_{i j} Z_{i j}=G_{N j}^{(k)}
$$

where $Z_{i j}$ is the amount of water diverted for irrigation at node $i$ in period $j$. The sum over $i$ runs over all the nodes in state $N$. Apart from minor modifications, the cG's strategy is as before. An initial feasible solution is chosen, which is improved upon by adjusting the $G_{N j}^{(k)}$ with contributions from the respective shadow prices.

If the scheme of algorithm II is used, this problem is even harder to solve than 
algorithm II. If the method adopted in algorithm III is the basis of the cG's actions, then complete decoupling may be achieved among the states. This happens because there is now only one constraint for each state. The input and output constraints that plagued the 'low flow quota' methods have been eliminated in favour of (1). If, for example, $G_{R A J}$ is reduced; but none of the other states' $G$ 's is changed, the extra water released by Rajasthan will manifest itself as increased flow at Farakka. This seems to be the most desirable feature of this method.

In order to minimize the chance of changing a $G$ in such a way that infeasibilities occur, the initial $G$ 's should be set high enough such that no water flows out from any one of the states. The situation is summarized in figure $3 \mathrm{~d}$.

The algorithm requires that the cG know the hydrological inputs to all the states. It can then ascertain that the states are not misrepresenting the amount of water they use by evaluating the following relationships;

$$
\sum_{i j} H_{i j}=\sum_{j} F_{N j}+\sum_{j} G_{N j}-\sum_{j} X_{N j}
$$

where $\sum_{i j} H$ is the annual hydrological input to the $N$ th state, $\sum_{j} F_{X j}$ is the amount of water flowing out of the state and $\sum_{j} X_{N j}$ is the input of water to the $N$ th state from its upstream neighbour.

After the cG chooses an initial allocation of $G_{N J}$ 's such that the constraints are met at Farakka, the SGS solve their problems and relay back to the CG their shadow prices on the $G_{N j}$, the ranges and their output $F_{N j}$. The reason for the cG's inability to determine the $F_{N j}$ a priori is because of the season-to-season coupling by groundwater pumping and the operating of reservoirs. This is precisely where the shortcomings of the algorithm lie. The cG is unable to determine, short of having the states do the calculations, how the seasonal output of UP, for example, will change if both $G_{R A}$ and $G_{U P}$ are changed. Because of this seemingly insurmountable difficulty it was decided not to investigate the method any further. It is conceivable, however, that because of the general stiffness of the Ganga model, the season-to-season coupling would not be strong enough to seriously interfere with the workings of the algorithm.

From a political point of view, the algorithm may not be a favourite of the sGs, as it may be construed to infringe on state autonomy.

\section{Computational experience}

\subsection{General remarks}

Calculations were made using the Harvard Business School's DEC 1070 system. The linear programming sub-problems were solved by the highly convenient and adaptable HPSLP subroutine available on this computer. Because of software, hardware, time and fiscal limitations, it was necessary to make a few simplifications in the original model to reduce the sizes of the sub-problems to solvable ones by the HBSLP program. Even so, the largest of the linear programs, that for UP, had 121 variables and 123 constraints. Admittedly, the input matrix was sparse, but such a large problem is still just about as big as the existing time-shared software can handle. The CG's master program was solved by hand. 


\subsection{Assumptions and simplifications}

Since in both the solutions presented by Rogers \& Kung (1985), the groundwater reserves were completely exploited at the end of the third season, it was decided to set the $D_{i j}$ 's to be identically zero $\left(D_{i j}\right.$ is the quantity of groundwater and irrigation drainage at node $i$ that is returned to the river at a downstream node). Further reductions in the size of UP's sub-problem were achieved by fixing the irrigation levels of nodes $13,24,33,44$ and 45 at the levels calculated by the previous two methods. The particular areas were chosen because their abundant water supplies assure their being irrigated at a constant level for not too drastic changes in $F_{\mathrm{RAJ}}$ and $F_{\mathrm{UP}}$.

Node 40 irrigates roughly equal areas in Madhya Pradesh and Rajasthan. Since power generation was not considered in the objectives of the states, it was assumed that the reservoirs at nodes 42 and 43 were operated in unison to derive the maximum irrigation benefits for both states.

Because of similar considerations up's reservoir at node 20 was operated in such a fashion as to aid the irrigation of Bihar. Of course if power generation were one of the objectives, operating policies would be drastically different.

\subsection{Notes on iterations}

Figure 5 represents the progress of the iterations for algorithms II and III.

5.3a Algorithm II: It proceeded smoothly until iteration four, at which point it was realized that a 'skip to another branch' was necessary if one was not to get stuck at a sub-optimal solution. This was done by increasing the first season output of uP from 3000 to $6500.8 \mathrm{~m}$ cu m, which was the limit dictated by the range of Bihar's shadow price for incoming water. This skip resulted in a slight decrease in the total area irrigated, but permitted a yet higher level to be reached in subsequent iterations. A similar procedure would have been performed after the ninth iteration, but as already pointed out earlier, the value of such a procedure at this stage would have been questionable. The optimization procedure was thus terminated at what is no doubt a sub-optimal solution. The value of the primal feasible feature of the algorithm is thus amply demonstrated. Figure 5 and table 1 summarize the status of the model after each iteration and indicate what changes were made from iteration to iteration.

Table 1. Algorithm II: Gross area irrigated (million hectares)

\begin{tabular}{|c|c|c|c|c|c|}
\hline Iteration & $\begin{array}{l}\text { Rajasthan } \\
\text { and M.P. }\end{array}$ & U.P & Bihar & West Bengal & Total \\
\hline 1 & $7 \cdot 42$ & 28.96 & 18.90 & $1 \cdot 11$ & $56 \cdot 39$ \\
\hline 2 & $7 \cdot 37$ & 29.08 & $19 \cdot 11$ & $1 \cdot 11$ & $56 \cdot 67$ \\
\hline 3 & $7 \cdot 38$ & $29 \cdot 22$ & $19 \cdot 12$ & $1 \cdot 11$ & $56 \cdot 83$ \\
\hline 4 & $7 \cdot 18$ & $29 \cdot 46$ & $19 \cdot 12$ & $1 \cdot 11$ & $56 \cdot 87$ \\
\hline 5 & $7 \cdot 18$ & 28.89 & $19 \cdot 68$ & $1 \cdot 11$ & $56 \cdot 86$ \\
\hline 6 & 7.02 & 29.07 & 19.68 & $1 \cdot 11$ & 56.88 \\
\hline 7 & 6.53 & $29 \cdot 58$ & $19 \cdot 68$ & $1 \cdot 11$ & 56.90 \\
\hline 8 & 6.36 & $29 \cdot 83$ & $19 \cdot 68$ & $1 \cdot 11$ & 56.98 \\
\hline 9 & $6 \cdot 33$ & 29.87 & 19.68 & $1 \cdot 11$ & 56.99 \\
\hline
\end{tabular}




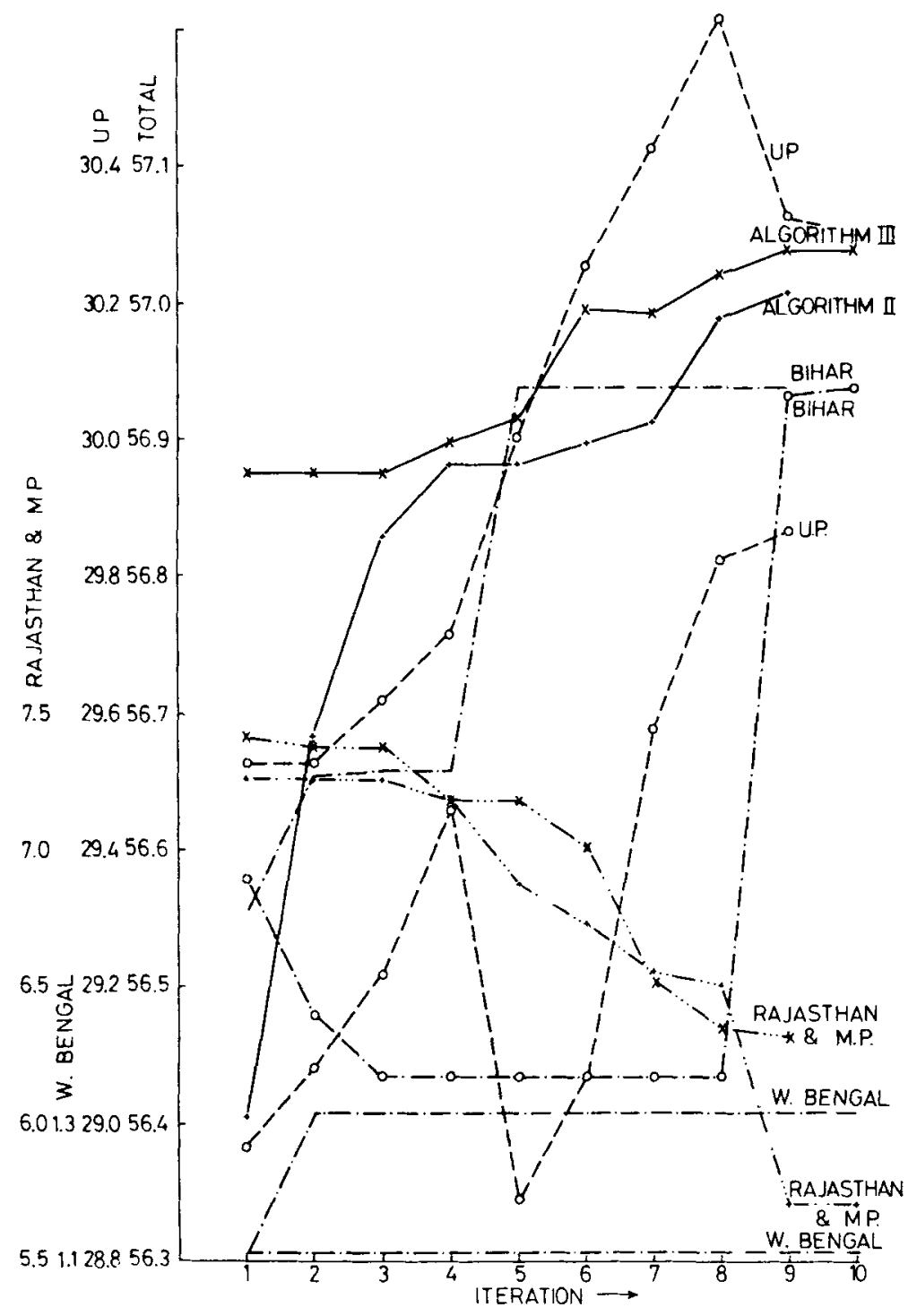

Figure 5. Iteration results of different algorithms.

5.3b Algorithm III It proceeded slowly and uneventfully. An effort was made at the eighth iteration to speed up convergence by increasing the third season input to uP by $2000 \mathrm{~m}$ cu $\mathrm{m}$ rather than the $679 \mathrm{~m}$ cu $\mathrm{m}$ that was indicated by the range on the shadow price. The attempt was a failure. The value of 2000 was promptly reduced to 679 in the ninth iteration. The progress of the algorithm and final results are summarised in table 2 and figure 5.

\section{Conclusions and discussions}

The foregoing discussion should illuminate the complications encountered when a 
Table 2. Algorithm III: Gross area irrigated (million hectares)

\begin{tabular}{rrrrrr}
\hline Iteration & $\begin{array}{l}\text { Rajasthan } \\
\text { and M.P. }\end{array}$ & U.P. & Bihar & West Bengal & Total \\
\hline 1 & 7.26 & 29.53 & 18.96 & 1.11 & 56.86 \\
2 & 7.26 & 29.53 & 18.76 & 1.32 & 56.87 \\
3 & 7.26 & 29.62 & 18.67 & 1.32 & 56.87 \\
4 & 7.18 & 29.72 & 18.67 & 1.32 & 56.89 \\
5 & 6.87 & 30.05 & 18.67 & 1.32 & 56.91 \\
6 & 6.73 & 30.26 & 18.67 & 1.32 & 56.98 \\
7 & 6.56 & 30.43 & 18.67 & 1.32 & 56.98 \\
8 & 6.51 & 30.52 & 18.67 & 1.32 & 57.02 \\
9 & 5.71 & 30.33 & 19.67 & 1.32 & 57.03 \\
10 & 5.71 & 30.31 & 19.68 & 1.32 & 57.02 \\
\hline
\end{tabular}

large river basin is decomposed into sub-problems that are serially coupled to each other. Both of the algorithms that were tested on the Ganga basin model seemed to work adequately, but their limitations, as discussed in $\S 3$, must never be lost sight of. It is not at all certain, for example, that algorithm II would lead to satisfactory solutions within a reasonable number of steps were it applied to a more flexible model than the one used here for the Ganga basin. As has been already suggested, a game-theoretical approach may be applicable here. Future studies should cover the behaviour of the resource allocation algorithm of $\S 4$, and should refine the model by including power generation in the objective functions. The effects of misrepresentation and how it may be precluded by the CG or how the CG can actually force the states to release a certain quantity of water to be used at Farakka should also be studied in detail.

One wonders whether algorithm II or algorithm III is the more socially equitable one. Were they equivalent computationally, the decision to use one or the other should be arrived at jointly by the central and state governments. As Kornai (1973) pointed out, it is unreasonable to expect real world planning processes to follow the confined ways and means of an elegant mathematical algorithm. While the results presented in this paper are numerically correct, the way in which they were arrived at, the iterative process between the CG and SGS, should not be taken too literally. There is so much that the algorithms ignore. Economic and political pressures, corruption, bribery, behindthe-scenes negotiations are all vastly more powerful forces in choosing what the actual allocations will be, than the naive decisions taken by our hypothetical cG based entirely on the computed shadow prices.

\section{References}

Chaturvedi M C, Rogers P, Kung S L 1985 Sadhana 8: 93-121

Kornai J 1973 Multilevel planning case studies in Mexico (eds) L M Goreux \& A S Manne (New York: North Holland) Chap. 5.3

Rogers P, Kung S L 1985 Sadhana 8: 123-134 